\title{
Du cadavre au robot - pourquoi les EPF se trompent
}

\section{Hans Stalder}

Prof. Dr med., membre de la rédaction

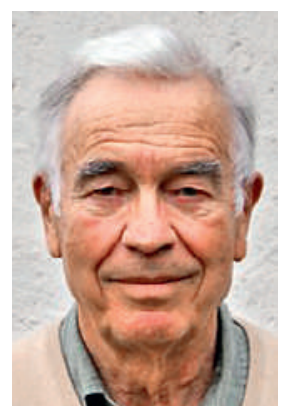

Quand je faisais mes études de médecine, il n'y avait que deux examens propédeutiques, et un examen fédéral à la fin des études. Zoologie et botanique figuraient comme disciplines du premier "propé» et l'anatomie jouait un rôle principal dans le deuxième. Le premier "être humain" qu'on rencontrait était un cadavre dans la salle d'autopsie. Et après chaque examen on se disait soulagé de pouvoir «oublier» tout ce qu'on avait appris. Depuis, les études de médecine ont été réformées et une réflexion approfondie a eu lieu sur les objectifs d'apprentissage. Il faut enseigner ce qui importe en médecine! Ainsi, zoologie et botanique ont été abandonnées et l'anatomie a perdu sa prédominance en faveur de la biologie, de l'épidémiologie, de l'éthique et d'autres sciences humaines. Dans certaines facultés, comme à Genève, les études ont été réorganisées de fond en comble, l'apprentissage par problème a été introduit pour les années précliniques, puis l'apprentissage à la résolution de problèmes en clinique. La formation ne se fait plus selon les disciplines, mais d'une façon intégrée. Une exposition clinique précoce (à Genève en deuxième année) a été réalisée: le premier «malade» n'est plus un cadavre, mais un patient à l'hôpital ou chez le médecin généraliste. Il fallait convaincre l'étudiant de l'importance de l'apprentissage des sciences de base en lui montrant leur application clinique.

L'introduction du système de Bologne avec le bachelor et le master n'a guère influencé ce concept: les études de médecine restent un ensemble de la première à la sixième année.

Maintenant cependant, cela devrait changer: l'Ecole polytechnique fédérale (EPF) de Zurich propose un bachelor en médecine basé sur les MINT: mathématiques, informatique, sciences naturelles et technologie. L'EPF de Lausanne s'y intéresse aussi.

A mon avis, il y a plusieurs raisons pour lesquelles cette proposition est mal pensée:

- «Dieser neue Studiengang entspricht vollumfänglich den Bedürfnissen des Standorts Nordwestschweiz mit seiner Life-Science-Industrie». C'est ainsi que s'est exprimée la rectrice de l'Université de Bâle (NZZ 12.9.2015). La formation des médecins ne doit pas répondre aux besoins de l'industrie, mais à ceux de la médecins praticiens. C'est dans ce but que le Conseil fédéral veut débloquer 100 millions de francs.

- En Suisse, c'est la pénurie de médecins de famille qui pèse le plus. Le soutien du Conseil fédéral à la formation des médecins devrait donc surtout combler ce manque. Mathématiques, informatique et technologie ne sont pas des domaines primordiaux pour l'exercice de la médecine de famille.

- Cela semble aussi être l'avis du peuple suisse. En acceptant un nouvel article constitutionnel sur la médecine de famille avec un score exceptionnel et un autre sur la médecine complémentaire, il n'exprime certainement pas le désir d'une médecine encore plus technique et informatisée!

- L'interdisciplinarité entre sciences précliniques et cliniques est un des acquis principaux des réformes des études de médecine. Un programme comme celui que proposent les EPF est un retour en arrière.

- Une exposition précoce à la clinique, qu'une école polytechnique ne pourra pas assurer, est un élément important pour stimuler l'apprentissage et pour éviter la démotivation des étudiants.

- Le goulet dans la formation des médecins ne se trouve pas au niveau du bachelor, où il est relativement facile d'augmenter le nombre des étudiants. Ce qui manque ce sont des places cliniques avec des patients, sans lesquelles la formation de médecins est inconcevable. Les 100 millions doivent donc être réservés à la formation clinique. Celle-ci peut être fournie principalement par les facultés de médecine actuelles liées à des hôpitaux universitaires et peut-être par de nouveaux sites - Fribourg, SaintGall, Lucerne, Tessin - et développée en ambulatoire par des cabinets médicaux.

Sans doute l'informatique et la technique jouent-elles et joueront-elles un rôle important dans le progrès de la médecine (et de l'industrie...). Une formation par des experts dans ce domaine est donc souhaitable. A mon avis elle doit se situer au niveau postgradué, sous forme d'un Master of Advanced Studies, et elle pourrait être fournie par les EPF. Nous tenons à l'acquis de l'exposition précoce à la médecine avec de vrais patients et n'aimerions pas que le premier «être humain» rencontré par l'étudiant devienne un robot. 